\title{
Microfluidic Analytical System with On-Line Luminol Chemiluminescence Detection Based on Annular Flow of Phase Separation Multiphase Flow
}

\author{
Junki Manai', Kei Nishiyama1, Kazuhiko Tsukagoshi1,2* \\ ${ }^{1}$ Department of Chemical Engineering and Materials Science, Faculty of Science and Engineering, Doshisha University, \\ Kyotanabe, Japan \\ ${ }^{2}$ Bio-Microfluidic Science Research Center, Doshisha University, Kyotanabe, Japan \\ Email: ${ }^{\star} k t s u k a g o @ m a i l . d o s h i s h a . a c . j p$
}

How to cite this paper: Manai, J., Nishiyama, K. and Tsukagoshi, K. (2017) Microfluidic Analytical System with OnLine Luminol Chemiluminescence Detection Based on Annular Flow of Phase Separation Multiphase Flow. Journal of Analytical Sciences, Methods and Instrumentation, 7, 29-39.

https://doi.org/10.4236/jasmi.2017.72003

Received: April 2, 2017

Accepted: June 9, 2017

Published: June 12, 2017

Copyright $\odot 2017$ by authors and Scientific Research Publishing Inc. This work is licensed under the Creative Commons Attribution International License (CC BY 4.0).

http://creativecommons.org/licenses/by/4.0/

\begin{abstract}
Microfluidic analytical system was developed based on annular flow of phase separation multiphase flow with a ternary water-hydrophilic/hydrophobic organic solvent solution. The analytical system was combined with on-line luminol chemiluminescence detection for catechin analysis. The water (10 $\mathrm{mM}$ phosphate buffer, $\mathrm{pH}$ 7.3)-acetonitrile-ethyl acetate mixed solution (3:8:4, volume ratio) containing $60 \mu \mathrm{M}$ luminol and $2 \mathrm{mM}$ hydrogen peroxide as a carrier was fed into the capillary tube (open-tubular fused-silica, $75 \mu \mathrm{m}$ inner diameter, $110 \mathrm{~cm}$ effective length) at a flow rate of $1.0 \mu \mathrm{L} \cdot \mathrm{min}^{-1}$. The carrier solution showed stable chemiluminescence as a baseline on the flow chart. Eight catechins were detected as negative peaks for their antioxidant potential with different detection times. The system was applied to analyze the amounts of catechin in commercially available green tea beverages.
\end{abstract}

\section{Keywords}

Microfluidic Analytical System, Phase Separation Multiphase Flow, Catechin, On-Line Luminol Chemiluminescence Detection

\section{Introduction}

Fluidic flow in a tube can be typically classified into one of two groups: homogeneous single-phase flows, which do not have a liquid-liquid interface, and immiscible multiphase flows, which have a liquid-liquid interface [1]. Electroosmotic and laminar flows generated in a microspace are basically classified 
as homogeneous single-phase flows. Over the last century, capillary electrochromatography [2], micellar electrokinetic chromatography [3], and hydrodynamic chromatography [4], were developed by manipulating electroosmotic and laminar flows. Various types of immiscible multiphase flows that generate liquid-liquid interfaces using water and hydrophobic organic solvents have been reported [5] [6]. These reports show droplet, slug, parallel, or annular flows depending on the conditions employed. Investigation concerning a liquid-liquid interface in microfluidic flow has been interesting and attractive in Lab-on-aChip and $\mu$-TAS research area [7] [8] [9]. However, to our knowledge, there have been no reports regarding capillary chromatography taking advantage of liquid-liquid interfaces based on such multiphase flows.

We have recently developed a method of generating a multiphase flow using two-phase separation mixed solvent solutions [10] [11] [12] [13]. When mixed solvent solutions, such as a water-hydrophilic/hydrophobic organic solvent solution, are delivered into a microspace, such as a capillary tube and a microchannel on a microchip, phase transformation of the mixed solvent solution occurs in the microspace with changes in temperature and/or pressure, leading to a kinetic liquid-liquid interface. These new types of multiphase flows are called "phase separation multiphase flows". We can also observe droplet, slug, parallel, and annular flows under different conditions in phase separation multiphase flow. We are particularly interested in annular flows, including inner and outer phases, generated in a phase separation multiphase flow. The specific microfluidic phenomenon and flow are termed the "tube radial distribution phenomenon" (TRDP) and "tube radial distribution flow" (TRDF), respectively [14] [15].

We have experimentally and theoretically investigated phase separation multiphase flows from the viewpoints of the mechanism of generation and functional appearance [14] [15] [16] [17]. Open-tubular capillary chromatography, designated as tube radial distribution chromatography (TRDC), has been developed based on the annular flow or TRDF from a phase separation multiphase flow. Various types of analyte were chromatographically separated through the opentubular capillary tube on the TRDC system, where the inner and outer phases in the TRDF act as the mobile and pseudostationary phases, respectively [14] [15].

In this study, we first examined luminol chemiluminescence (CL) behavior in a ternary mixed solvent solution of water-acetonitrile-ethyl acetate, and the mixed solvent solution was found to enhance luminol CL in time and intensity under certain conditions. Based on these new experimental data, we combined the TRDC system with on-line luminol CL detection for catechin analysis. The present fluidic analytical system was used to detect eight catechins with each detection times on the CL profile and further analyze the amounts of catechin in commercially available green tea beverages.

\section{Experimental}

\subsection{Reagents and Materials}

Water was purified with an Elix UV 3 system (Millipore Co., Billerica, MA). All 
reagents used were commercially available and of analytical grade. Luminol, hydrogen peroxide, acetonitrile, ethyl acetate, antioxidant reagents, and eight catechins as well as catechin mixture from green tea were purchased from Wako Pure Chemical Industries, Ltd. (Osaka, Japan). Fused-silica capillary tubes with an inner diameter of $75 \mu \mathrm{m}$ were purchased from GL Science Co. (Tokyo, Japan).

\subsection{Measurement in a Batch System}

The CL profile was examined with a batch-type detection system (CLA-FS4; Tohoku Electronic Industrial Co. Ltd., Sendai, Japan). To the water (10 mM phosphate buffer, $\mathrm{pH}$ 7.3)-acetonitrile-ethyl acetate mixed solution (3:8:4, volume ratio; $2.5 \mathrm{~mL}$ ) containing $0.1 \mu \mathrm{M}$ luminol in a petri dish was added $15 \mathrm{mM}$ hydrogen peroxide solution $(50 \mu \mathrm{L})$ with constant stirring to obtain the CL profile. To examine the suppressive effects of various antioxidant reagents on CL, antioxidant reagents dissolved in ternary mixed solvent solution $(250 \mu \mathrm{M}, 0.1$ $\mathrm{mL}$ ) were added to the solutions in petri dishes that emitted around the maximum CL intensity, and the decreases in CL intensities due to their antioxidant potential were measured.

\subsection{Fluidic Analytical System with On-Line CL Detection}

Figure 1 shows a schematic diagram of the present analytical system with online CL detection, comprised of an open-tubular fused-silica capillary tube (75 $\mu \mathrm{m}$ inner diameter and $140 \mathrm{~cm}$ length (effective length, $110 \mathrm{~cm}$ )), a micro-syringe pump (MF-9090; Bioanalytical Systems, Inc., West Lafayette, IN), and a CL detector (Kimoto Electronic Co. Osaka, Japan). The tube temperature was controlled by dipping the capillary tube in water maintained at a definite temperature in a beaker with stirring. Water (10 mM phosphate buffer, $\mathrm{pH}$ 7.3)-acetonitrilel-ethy acetate mixture (3:8:4 volume ratio) solution containing $60 \mu \mathrm{M} \mathrm{lu}$ minol and $2 \mathrm{mM}$ hydrogen peroxide was used as a carrier solution. Analyte solutions were prepared with the carrier solution.

The analyte solution was introduced directly into the capillary inlet side by the gravity method $(20 \mathrm{~cm}$ height for $15 \mathrm{~s})$. After analyte injection, the capillary inlet was connected through a joint to a micro-syringe. The syringe was placed on the micro-syringe pump. The carrier solution was fed into the capillary tube at a

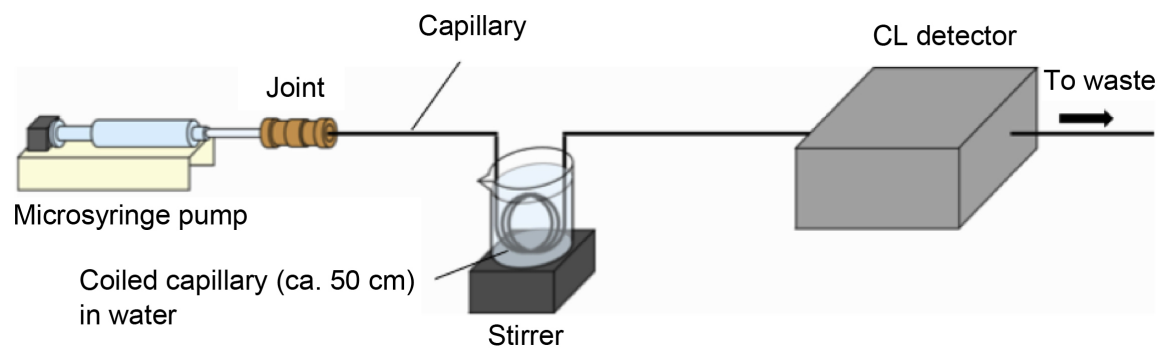

Figure 1. Schematic diagram of the present microfluidic analytical system with on-line CL detection. 
flow rate of $1.0 \mu \mathrm{L} \cdot \mathrm{min}^{-1}$ under laminar flow conditions. Negative peaks observed from the CL baseline were detected by the CL detector.

\section{Results and Discussion}

\subsection{Profile with the Ternary Mixed Solvent Solution}

The phase diagram of water (10 mM phosphate buffer, $\mathrm{pH}$ 7.3)-acetonitrile-ethyl acetate is shown in Figure 2; the dotted line indicates the boundary or solubility curve. The CL profiles were examined for ternary mixed solvent solutions with (a) organic solvent-rich and (b) water-rich component ratios, with a batch-type CL measurement system. Mixed solutions of (a) and (b) are most commonly used as carrier solutions in the TRDC system [14] [15]. In the TRDC system the specific homogeneous mixed solutions are required for generating TRDF; the component ratios of them are positioned near the boundary curve on the phase diagram, such as (a) and (b), and when they become away from the curve, TRDF cannot be observed because phase transformation does not occurs. The obtained CL profiles are shown in Figure 3, taken together with the CL profile obtained with $10 \mathrm{mM}$ phosphate buffer ( $\mathrm{pH}$ 7.3) solution. From the obtained CL profiles, the component ratio (a) showed the greater and longer lasting CL, and was selected as the carrier solution for use in the system. It was completely confirmed that the CL lasted more than 8 hour with good reproducibility. There was little information of CL in the ternary water-hydrophilic/hydrophobic organic solvent mixed solution, and the reason why the ternary mixed solution enhanced CL is not yet clear. However, the ternary homogeneous solution near the boundary

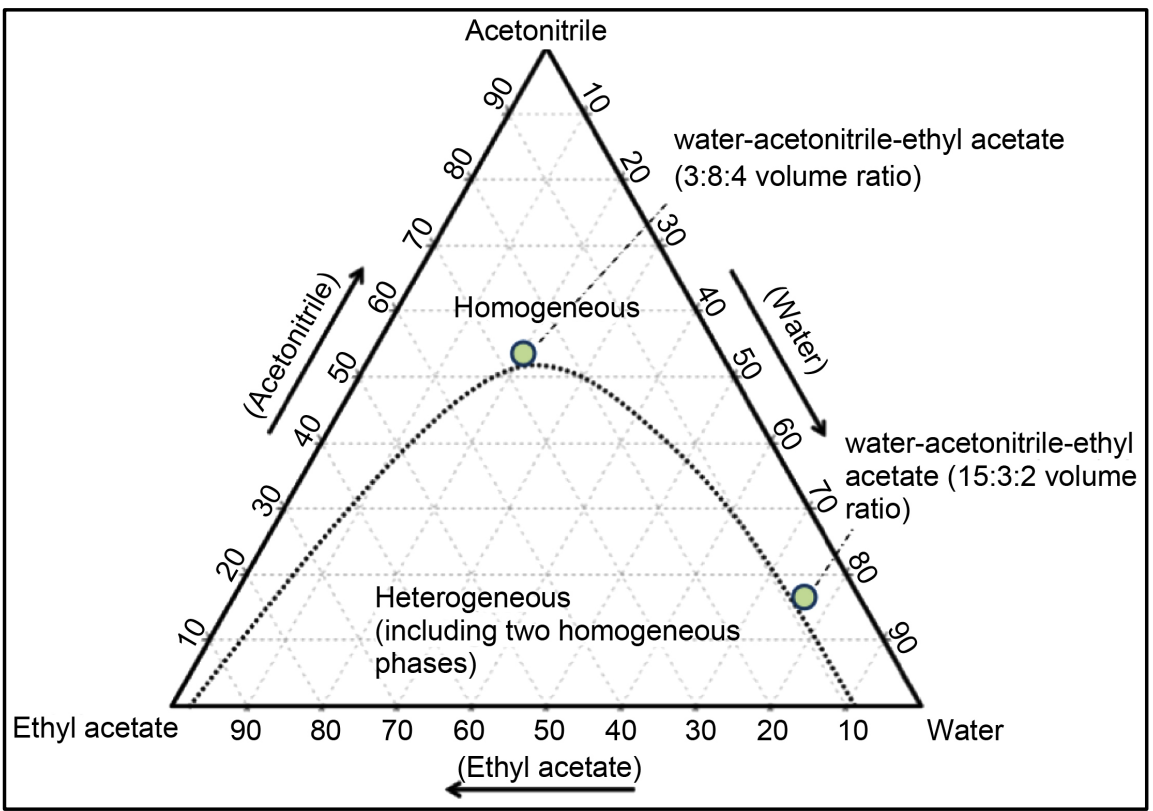

Figure 2. Phase diagram for the ternary water-acetonitrile-ethyl acetate mixed solvent solution. The dotted curve in the diagram indicates the homogeneous-heterogeneous solution boundary curve. The component ratios of the solvents, (a) and (b), are water (10 $\mathrm{mM}$ phosphate buffer $\mathrm{pH}$ 7.3)-acetonitrile-ethyl acetate of 3:8:4 and 15:3:2 volume ratio, respectively. 


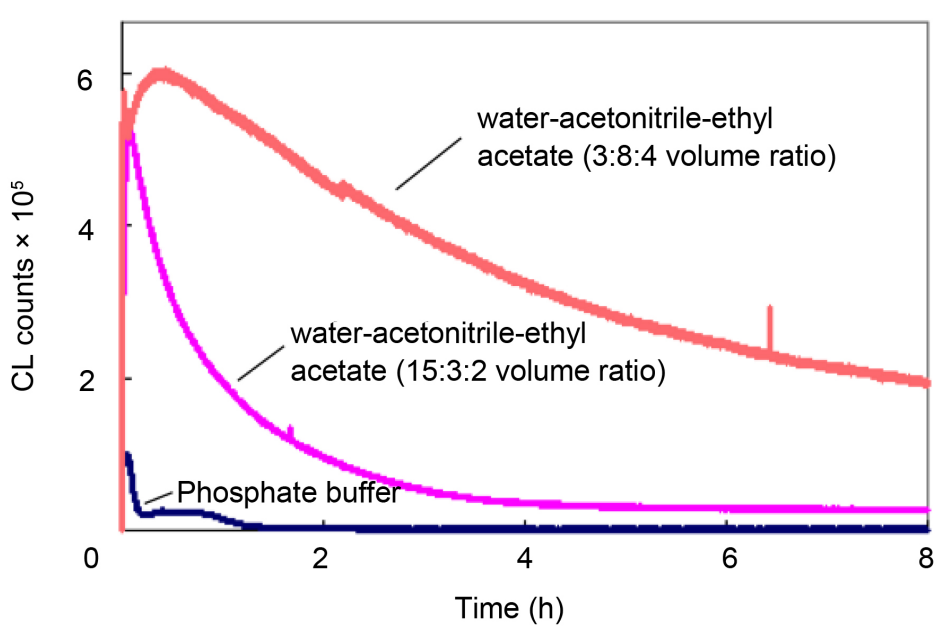

Figure 3. CL profiles obtained with the batch-type CL detector. To 0.1 $\mu \mathrm{M}$ luminol solution $(2.5 \mathrm{~mL})$ prepared with (a) water $(10 \mathrm{mM}$ phosphate buffer, $\mathrm{pH} 7.3$ )-acetonitrile-ethyl acetate (3:8:4 volume ratio), (b) water (10 mM phosphate buffer, $\mathrm{pH}$ 7.3)-acetonitrile-ethyl acetate (15:3:2 volume ratio), or (c) $10 \mathrm{mM}$ phosphate buffer ( $\mathrm{pH} 7.3$ ) was added $15 \mathrm{mM}$ hydrogen peroxide solution $(50 \mu \mathrm{L})$ prepared with $10 \mathrm{mM}$ phosphate buffer ( $\mathrm{pH} 7.3)$.

curve may cause formation of small clusters (micro-clusters) in the solvent solution. The interface of the clusters, with features of hydrophilic and hydrophobic interfaces, may be effective as a CL reaction domain.

\subsection{Effects of Antioxidants on CL Profiles}

The effects of antioxidants on the CL profiles were examined using a batch-type CL measurement system. The analytical conditions were described in the Experimental section. 2-Propanol and nordihydroguaiaretic acid that acted as an antioxidant for hydroxyl radicals (.OH) decreased the CL intensity by $80 \%$ and $100 \%$, respectively, compared to the maximum CL intensity in the CL profiles. Other antioxidants, ascorbic acid for superoxide radical anion $\left(\cdot \mathrm{O}_{2}^{-}\right)$, singlet oxygen $\left({ }^{1} \mathrm{O}_{2}\right)$, and hydrogen peroxide $\left(\mathrm{H}_{2} \mathrm{O}_{2}\right)$, sodium azide for ${ }^{1} \mathrm{O}_{2}$, and nitrobluetetrazolium for $\cdot \mathrm{O}_{2}^{-}$, did not decrease $\mathrm{CL}$ intensity. These observations indicated that $\cdot \mathrm{OH}$ mainly acted as an oxidant for the luminol CL reaction in the present ternary mixed solvent solution. The researchers have been interested in $\cdot \mathrm{OH}$ action in CL reaction and CL analysis [18] [19]. Generally, $\cdot \mathrm{OH}$ has a very short life time and is one of the strongest oxidants. It is interesting that $\cdot \mathrm{OH}$ was generated gradually from the present mixed solvent solution.

\subsection{TRDF Observation}

We confirmed the TRDF, where the organic solvent-rich major inner phase (perylene, blue) and the water-rich minor outer phase (Eosin Y, green) were generated, in the capillary tube of the system using a fluorescence microscope-CCD camera. The obtained fluorescence photograph is shown in Figure 4; analytical conditions are also described in the figure legend. Phase separation multiphase 


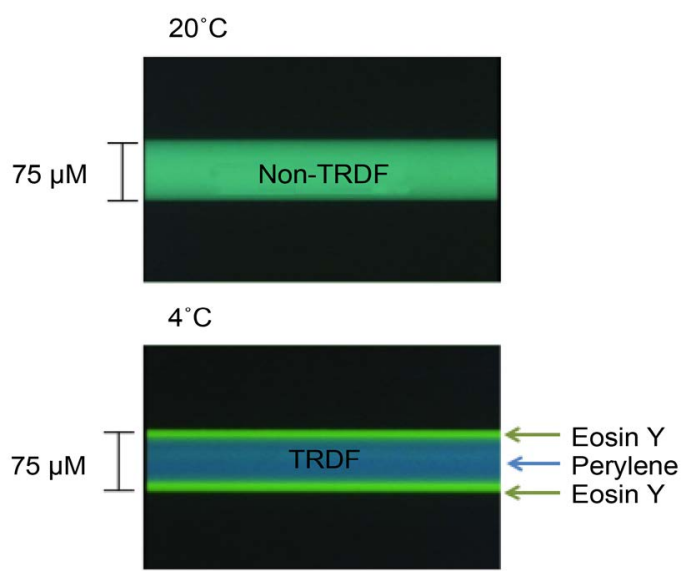

Figure 4. Fluorescence photograph of TRDF observed with a fluorescence microscope-CCD camera system. (a) Non-TRDF at $20^{\circ} \mathrm{C}$ and (b) TRDF at $5^{\circ} \mathrm{C}$. Conditions: Capillary tube, $140 \mathrm{~cm}$ (observation point: 110 $\mathrm{cm}$ from the inlet) of $75 \mu \mathrm{m}$ i.d. fused silica; carrier, water (10 $\mathrm{mM}$ phosphate buffer, $\mathrm{pH}$ 7.2)-acetonitrile-ethyl acetate (3:8:4 volume ratio) containing 0.1 $\mathrm{mM}$ perylene and $1 \mathrm{mM}$ Eosin $\mathrm{Y}$, and flow rate, 1.0 $\mu \mathrm{L} \cdot \mathrm{min}^{-1}$.

flow occurred with the ternary mixed solvent solution in the capillary tube at lower temperature through phase transformation; the annular flow or TRDF as shown in the figure could be observed under certain conditions.

\subsection{Fluidic Analytical System with On-Line CL Detection}

We developed various types CL detection system for flow-injection analysis, high-performance liquid chromatography, and capillary electrophoresis [20] [21]. However, all were off-line CL detection systems, where it was necessary to mix the eluate with reagent at the end of the tube or capillary. To our knowledge, there have been no reports regarding an on-capillary CL detection system. In this study, luminol CL reaction was shown to continue for several hours, maintaining sufficient CL with the ternary mixed solvent solution. The CL phenomenon could lead to the development of on-capillary CL detection systems without a mixing process at the end of the capillary, where analytes as inhibitors for $C L$ reaction are detected as negative peaks from the baseline $C L$ intensity.

The antioxidant reagents were subjected to the system with on-line CL detection. 2-Propanol and nordihydroguaiaretic acid were detected with negative peaks due to their antioxidant potential for luminol CL, but other antioxidants (superoxide radical anion, sodium azide, and nitrobluetetrazolium) were not different from baseline. The CL quenching tendency in the system was consistent with that observed with the batch-type CL detector mentioned above.

\subsection{Chromatographic Information of Catenchin on Fluidic Analytical System}

Catechin is a well-known antioxidant, and is one of the polyphenol derivatives, 
where the hydroxyl groups work as antioxidant-sites, that react with radical species, such as hydroxyl radicals. Eight catechins were analyzed with the system, and all showed the negative peaks. The catechin structures were classified according to the number of hydroxyl groups containing diol and triolstructures. The relative CL peak areas as negative peaks are summarized with the numbers of hydroxyl groups in Table 1 . As shown here, the negative CL peak areas increased with increasing number of hydroxyl groups.

The elution times of catechin became later with increasing hydrophilicity expressed as $\log \mathrm{P}$ (octanol/water partition coefficient) [22] and $\log \mathrm{K}$ (distribution coefficient) (Table 2). The value of $\log \mathrm{P}$ acts as an index of hydrophobicity. The value of $\mathrm{K}$ was examined as a distribution coefficient of upper phase (organic solvent-rich) and lower phase (water-rich) for each catechin that was obtained through phase transformation of the ternary mixed solvent solution in a batch vessel. The values of both increased with increasing hydrophobicity of the compounds.

In the present system, the organic solvent-rich major inner phase acts as a mobile phase, while the water-rich minor outer phase acts as a pseudostationary phase. That is, as the outer phase moves very slowly according to parabolic flow

Table 1. Relative CL peak areas as negative peaks for catechins with diol and/or triol hydroxyl groups estimated based on 100 for epigallocatechingallate.

\begin{tabular}{cccc}
\hline \multirow{2}{*}{ Antioxidants } & \multicolumn{2}{c}{ Hydroxyl group } & \multirow{2}{*}{ Relative CL peak area } \\
\cline { 2 - 3 } & Diol & Triol & 64.0 \\
Catechin & 1 & 0 & 48.0 \\
Epicatechin & 1 & 0 & 76.8 \\
Gallocatechin & 0 & 1 & 81.6 \\
Epigallocatechin & 0 & 1 & 92.8 \\
Catechin gallate & 1 & 1 & 91.0 \\
Epicatechin gallate & 1 & 1 & 96.0 \\
Gallocatechin gallate & 0 & 2 & 100 \\
Epigallocatechin gallate & 0 & 2 & \\
\hline
\end{tabular}

Table 2. Detection times of CL peaks for catechins with values of $\log P$ and $\log$.

\begin{tabular}{cccc}
\hline Antioxidants & LogP & LogK & Detection Time/min \\
\hline Catechin & 0.31 & -0.11 & 6.2 \\
Epicatechin & 0.11 & -0.17 & 6.2 \\
Gallocatechin & -0.32 & -0.44 & 6.3 \\
Epigallocatechin & -0.50 & -0.55 & 6.5 \\
Catechin gallate & 1.55 & 1.03 & 5.5 \\
Epicatechin gallate & 1.06 & 0.88 & 5.7 \\
Gallocatechin gallate & 0.92 & 0.79 & 5.8 \\
Epigallocatechin gallate & 0.39 & 0.47 & 6 \\
\hline
\end{tabular}


under laminar flow conditions, the outer phase acts as a pseudohydrophilic-stationary phase on chromatographic separation mode. The hydrophilic compounds were distributed into the outer phase or pseudohydrophilic-stationary phase, and then eluted slowly, as shown in Table 2. Figure 5 shows the separation of catechingallate and epigallocatechin with the system. Catechingallate had the largest values of $\log \mathrm{P}$ and $\log \mathrm{K}$, and epigallocatechin had the smallest values. Naturally, catechingallate eluted earlier than epigallocatechin, as shown in Figure 5. They were separated and detected with a base-line separation. The TRDF has been reported in a microspace, such as a capillary tube and a microchannel on a microchip, with various types of two-phase separation mixed solutions [14] [15]. The present system has a potential as chromatographic instrument, which was described as TRDC in the introduction section. The system will improved to separate more several compounds chromatographically in the future.

\subsection{Catechin Analysis}

We examined catechin (catechin mixture from green tea) using the present system. The most common species of catechin included in green tea is epigallocatechingallate, which usually accounts for more than $40 \%$ of the total amount of catechin. Although the catechin mixture from green tea was not separated by the present system under the conditions used, a negative CL peak was observed on the flow profile at around 7 minutes.

We attempted to estimate the total amounts of catechin in commercial green tea beverages using the present system. First, the relationship between the catechin concentration of the above catechin mixture from green tea and the percentage

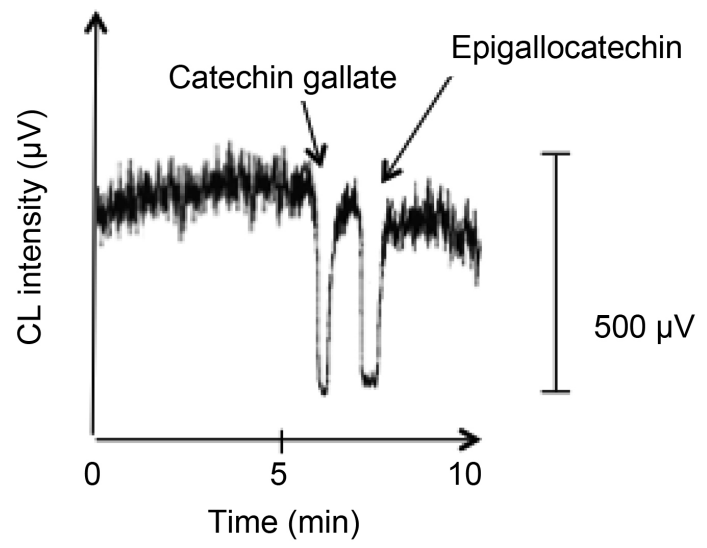

Figure 5. Flow profile of a mixture of catechingallate and epigallocatechin obtained by the microfluidic analytical system with on-line CL detection. Conditions: Capillary tube, $140 \mathrm{~cm}$ (effective length: $110 \mathrm{~cm}$ ) of $75 \mu \mathrm{m}$ i.d. fused silica; carrier, water $(10 \mathrm{mM}$ phosphate buffer, $\mathrm{pH}$ 7.3)-acetonitrile-ethyl acetate (3:8:4 volume ratio) containing $60 \mu \mathrm{M}$ luminol and $2 \mathrm{mM}$ hydrogen peroxide; sample injection, $20 \mathrm{~cm}$ height (gravity) $\times 15 \mathrm{~s}$; flow rate, $1.0 \mu \mathrm{L} \cdot \mathrm{min}^{-1}$, tube temperature, $15^{\circ} \mathrm{C}$; and catechin concentration, $250 \mu \mathrm{M}$ each. 
inhibition due to the negative CL peak was examined. The molecular weight of epigallocatechingallate (458.37) was used as that of the mixture. The negative CL peak responded over the range of $0.05-1 \mathrm{mM}$ catechin with a detection limit of $0.05 \mathrm{mM}(\mathrm{S} / \mathrm{N}=3)$. A linear relationship was observed for the concentration range of $0.05-1 \mathrm{mM}$ (correlation coefficient, 0.985 ). Tenfold-diluted commercial green tea beverage samples were analyzed using this system. Negative CL peaks were also observed on the CL profile at a detection time of ca. 7 minutes. Using the above-mentioned calibration curve, the commercial green teas, A, B, and $C$, were found to include catechin at concentrations of 363,186 , and 116 $\mu \mathrm{M}$, (average values from 4 - 7 measurements), respectively. The data obtained with the present method were consistent with the manufacturers' reported catechin concentrations for $A, B$, and $C(337,175$, and $109 \mu \mathrm{M}$, respectively).

\section{Conclusions}

Microfluidic analytical system was developed with CL detection based on TRDF from phase separation multiphase flow. The simple microfluidic system consisted of single delivery line with on-capillary CL detection. The ternary mixed solvent solution of water-acetonitrile-ethyl acetate (3:8:4 volume ratio) was used as the carrier solution, because it was shown to act as an enhancer of the luminol-hydrogen peroxide CL reaction. The antioxidants for hydroxyl radicals, 1,2-propanol and nordihydroguaiaretic acid, quenched the CL intensity. Eight catechins that acted as antioxidants for hydroxyl radicals were detected as negative CL peaks due to their antioxidant potential with different detection times on the flow profile. The system could estimate the amounts of catechins in commercially available green tea.

\section{Acknowledgements}

This work was supported by a Grant-in-Aid for Scientific Research (B) from the Ministry of Education, Culture, Sports, Science, and Technology, Japan (MEXT) (No. 17H03083).

\section{References}

[1] Bird, R.B., Stewart, W.E. and Lightfoot, E.N. (2002) Shell Momentum Balances and Velocity Distribution in Laminar Flow. Transport Phenomena, Wiley, Toronto.

[2] Tsuda, T. (1987) Electrochromatography Using High Applied Voltage. Analytical Chemistry, 59, 521-523. https://doi.org/10.1021/ac00130a032

[3] Terabe, S., Otsuka, K., Ichikawa, K., Tsuchiya, A. and Ando, T. (1984) Electrokinetic Separations with Micellar Solutions and Open-Tubular Capillaries. Analytical Chemistry, 56, 111-113. https://doi.org/10.1021/ac00265a031

[4] Small, H. (1974) Hydrodynamic Chromatography. A Technique for Size Analysis of Colloidal Particles. Journal of Colloid and Interface Science, 48, 147-161. https://doi.org/10.1016/0021-9797(74)90337-3

[5] Jovanovic, J., Rebrov, E.V., Nijhuis, T.A.X., Kretzer, M.T., Hessel, V. and Schouten, J.C. (2012) Liquid-Liquid Flow in a Capillary Microreactor: Hydrodynamic Flow Patterns and Extraction Performance. Industrial \& Engineering Chemistry Re- 
search, 51, 1015-1026. https://doi.org/10.1021/ie200715m

[6] Ami, T., Awata, K., Umekawa, H. and Ozawa, M. (2012) Flow Characteristics of Oil-Water Mixture in Horizontal Mini-Channel (Influence of Inner Diameter and Geometry of Mixer). Japanese Journal of Multiphase Flow, 26, 302-311. https://doi.org/10.3811/jjmf.26.302

[7] Yang, W., Lee, K.K. and Choi, S. (2017) A Laminar-Flow Based Microbial Fuel Cell Array. Sensors and Actuators, B: Chemical, 243, 292-297. https://doi.org/10.1016/j.snb.2016.11.155

[8] Li, J., Mittal, N., Mak, S.Y., Song, Y. and Shum, H.C. (2015) Perturbation-Induced Droplets for Manipulating Droplet Structure and Configuration in Microfluidics. Journal of Micromechanics and Microengineering, 25, 084009/1-084009/12. https://doi.org/10.1088/0960-1317/25/8/084009

[9] Sinzato, Y.Z., Sousa, D., Nuno Jorge, J. and Francisco, R. (2017) An Experimental Investigation of the Interfacial Tension Between Liquid-Liquid Mixtures in the Presence of Surfactants. Experimental Thermal and Fluid Science, 85, 370-378. https://doi.org/10.1016/j.expthermflusci.2017.03.011

[10] Jinno, N., Itano, M., Hashimoto, M. and Tsukagoshi, K. (2009) Capillary Chromatography Based on Tube Radial Distribution of Aqueous-Organic Mixture Carrier Solvents. Talanta, 79, 1348-1353. https://doi.org/10.1016/j.talanta.2009.06.001

[11] Murakami, M., Jinno, N., Hashimoto, M. and Tsukagoshi, K. (2011) Tube Radial Distribution Phenomenon of Ternary Mixed Solvents in a Microspace under Laminar Flow Conditions. Analytical Sciences, 27, 793-798.

https://doi.org/10.2116/analsci.27.793

[12] Jinno, N., Murakami, M., Mizohata, K., Hashimoto, M. and Tsukagoshi, K. (2011) Fluorescence Observation Supporting Capillary Chromatography Based on Tube Radial Distribution of Carrier Solvents under Flow Conditions. Analyst, 135, 927 932. https://doi.org/10.1039/C0AN00820F

[13] Nagatani, K., Shihata, Y., Matsushita, T. and Tsukagoshi, K. (2016) Tube Radial Distribution Flow Separation in a Microchannel Using an Ionic Liquid Aqueous Two-Phase System Based on Phase Separation Multi-Phase Flow. Analytical Sciences, 32, 1371-1374. https://doi.org/10.2116/analsci.32.1371

[14] Tsukagoshi, K. (2015) Investigation of Specific Microfluidic Flow with Two-Phase Separation Mixed Solvent Solutions and Application to Flow Technology. Journal of Flow Injection Analysis, 32, 89-95.

[15] Tsukagoshi, K. (2014) Fundamental Research and Application of the Specific Fluidic Behavior of Mixed Solvents in a Microspace. Analytical Science, 30, 65-73. https://doi.org/10.2116/analsci.30.65

[16] Fujinaga, S., Hashimoto, M., Tsukagoshi, K. and Mizushima, J. (2015) Consideration of Tube Radial Distribution Phenomenon under Laminar Flow Conditions Based on the Weber Number. Journal of Chemical Engineering of Japan, 48, 947952. https://doi.org/10.1252/jcej.15we039

[17] Fujinaga, S., Hashimoto, M., Tsukagoshi, K. and Mizushima, J. (2016) Consideration of Inner and Outer Phase Configuration in Tube Radial Distribution Phenomenon Based on Viscous Dissipation in a Microfluidic Flow Using Various Types of Mixed Solvent Solutions. Analytical Sciences, 32, 455-461. https://doi.org/10.2116/analsci.32.455

[18] Shah, S.N.A., Zheng, Y., Li, H. and Lin, J.-M. (2016) Chemiluminescence Character of ZnS Quantum Dots with Bisulphite-Hydrogen Peroxide System in Acidic Medium. Journal of Physical Chemistry C, 120, 9308-9316.

https://doi.org/10.1021/acs.jpcc.6b01925 
[19] Cao, Y., Sui, D., Zhou, W. and Lu, C. (2016) Highly Selective Chemiluminescence Detection of Hydroxyl Radical via Increased-Electron Densities of Rhodamine B on Montmorillonite Matrix. Sensors and Actuators, B: Chemical, 225, 600-606.

[20] Hara, T. and Tsukagoshi, K. (1990) Chemiluminescence Analysis of Biological Constituents Using Metal-Complex Catalysts. A Review. Analytical Sciences, 6, 797 806. https://doi.org/10.2116/analsci.6.797

[21] Tsukagoshi, K., Nakahama, K. and Nakajima, R. (2004) Direct Detection of Biomolecules in a Capillary Electrophoresis-Chemiluminescence Detection System. Analytical Chemistry, 76, 4410-4415. https://doi.org/10.1021/ac030344i

[22] Shoji, A., Yanagida, A., Shindo, H. and Shibusawa, Y. (2004) Comparison of Elution Behavior of Catechins in High-Performance Liquid Chromatography with That on High-Speed Countercurrent Chromatography. Bunseki Kagaku, 53, 953.

https://doi.org/10.2116/bunsekikagaku.53.953

\section{Submit or recommend next manuscript to SCIRP and we will provide best} service for you:

Accepting pre-submission inquiries through Email, Facebook, LinkedIn, Twitter, etc. A wide selection of journals (inclusive of 9 subjects, more than 200 journals)

Providing 24-hour high-quality service

User-friendly online submission system

Fair and swift peer-review system

Efficient typesetting and proofreading procedure

Display of the result of downloads and visits, as well as the number of cited articles

Maximum dissemination of your research work

Submit your manuscript at: http://papersubmission.scirp.org/

Or contact jasmi@scirp.org 